\title{
Evolución de la resistencia antimicrobiana de bacilos Gram negativos en unidades de cuidados intensivos en Colombia
}

Cristhian Hernández-Gómez, Víctor M. Blanco, Gabriel Motoa, Adriana Correa, Juan José Maya, Elsa de la Cadena, Marcela Perengüez, Laura Rojas, Alejandra Hernández, Marta Vallejo, María Virginia Villegas, Grupo de Resistencia Bacteriana Nosocomial en Colombia

Centro Internacional de Entrenamiento e Investigaciones Médicas (CIDEIM), Cali, Colombia.

Introducción. La evolución de la resistencia bacteriana constituye una amenaza para la salud pública mundial. Los sistemas de vigilancia epidemiológica han integrado técnicas de biología molecular para mejorar las estrategias de control.

Objetivo. Describir los perfiles moleculares y fenotípicos de los bacilos Gram negativos en unidades de cuidados intensivos de 23 hospitales de Colombia entre 2009 y 2012.

Materiales y métodos. Se diseñó un estudio descriptivo en 23 hospitales del Grupo para el Estudio de la Resistencia Nosocomial (sic.) en Colombia. Se analizaron 38.048 aislamientos usando WHONET durante el periodo descrito. Se describieron perfiles de resistencia para Escherichia coli, Klebsiella pneumoniae, Pseudomonas aeruginosa y Acinetobacter baumannii. En 1.248 cepas se realizó reacción en cadena de la polimerasa (PCR) para detectar las carbapenemasas clínicamente más relevantes.

Resultados. Escherichia coli fue el microorganismo más frecuente (promedio=14,8 \%); la frecuencia de aislamientos de K. pneumoniae aumentó de $11 \%$ en 2009 a $15 \%$ en 2012 (p<0,001). La tendencia de los perfiles de multirresistencia aumentó en todas las especies estudiadas. De los aislamientos de $K$. pneumoniae evaluados, 68,4 \% fue positivo para KPC (Klebsiella pneumoniae Carbapenemase), mientras que la VIM (Verona Integron-encoded Metallo-betalactamase) en $P$. aeruginosa se observó en 46,5\%.

Conclusiones. Se observó un incremento en la tendencia de los microorganismos hacia la multirresistencia y una amplia distribución de las carbapenemasas. La articulación de la biología molecular con los sistemas de vigilancia permitió integrar el análisis del fenotipo con los mecanismos de resistencia involucrados en las bacterias estudiadas. Este análisis permitirá la elaboración de guías para el uso adecuado de antimicrobianos y contribuirá a la contención de estas bacterias multirresistentes en Colombia.

Palabras clave: bacterias Gram negativas, farmacorresistencia bacteriana, vigilancia epidemiológica, Colombia.

doi: http://dx.doi.org/10.7705/biomedica.v34i0.1667

Evolution of antimicrobial resistance in Gram negative bacilli from intensive care units in Colombia

Introduction: The continuous evolution of antimicrobial resistance poses a major threat to public health worldwide. Molecular biology techniques have been integrated to epidemiological surveillance systems to improve the control strategies of this phenomenon.

Objective: To describe the phenotypic and molecular profiles of the most important Gram negative bacilli from intensive care units in 23 Colombian hospitals during the study period 2009-2012.

Materials and methods: A descriptive study was conducted in 23 hospitals belonging to the Colombian Nosocomial Resistance Study Group. A total of 38.048 bacterial isolates were analyzed using WHONET over a four-year period. The antimicrobial resistant profiles were described for Escherichia coli, Klebsiella pneumoniae, Pseudomonas aeruginosa, and Acinetobacter baumannii. Polymerase chain reaction was performed in 1.248 strains to detect the most clinically relevant carbapenemases.

Results: Escherichia coli was the most frequently isolated organism (mean=14.8\%). Frequency of K. pneumoniae increased significantly from $11 \%$ in 2009 to $15 \%$ in $2012(p<0.001)$. All screened isolates had rising trends of multidrug-resistant profiles. KPC (Klebsiella pneumoniae carbapenemase)

\section{Contribución de los autores:}

Cristhian Hernández-Gómez, Víctor M. Blanco y Gabriel Motoa: análisis e interpretación epidemiológica de los datos. Adriana Correa, Juan José Maya, Elsa de la Cadena, Marcela Perengüez, Laura Rojas, Alejandra Hernández, Marta Vallejo y el Grupo de Resistencia Bacteriana Nosocomial en Colombia: análisis e interpretación de la epidemiología molecular de los aislamientos. María Virginia Villegas: dirección científica, interpretación de los datos.

Todos los autores participaron en la escritura y aprobación final del manuscrito. 
was detected in $68.4 \%$ of $K$. pneumoniae isolates while VIM (Verona integron-encoded metallobetalactamase) was present in $46.5 \%$ of them.

Conclusion: In this study, an increase in the trend of multidrug-resistant organisms and a wide distribution of carbapenemases was observed. The integration of molecular biology to surveillance systems allowed the compilation of this data, which will aid in the construction of guidelines on antimicrobial stewardship for prevention in Colombia.

Key words: Gram-negative bacteria; drug resistance, bacterial; epidemiological surveillance, Colombia. doi: http://dx.doi.org/10.7705/biomedica.v34i0.1667

Las infecciones causadas por microorganismos multirresistentes se asocian con estancias hospitalarias prolongadas, mayores tasas de fracaso terapéutico, aumento en la mortalidad, y un incremento en los costos derivados de la atención clínica que amenaza la sostenibilidad de cualquier sistema de salud $(1,2)$. Esta problemática mundial tiene un mayor impacto en hospitales de alta complejidad por el gran número de pacientes críticamente enfermos, de huéspedes inmunocomprometidos, la presencia de múltiples enfermedades concomitantes y el uso frecuente de dispositivos invasivos (3). Entre los factores más importantes relacionados con la selección y diseminación de bacterias multirresistentes están el uso inapropiado de antibióticos (4) y la aplicación ineficiente de las medidas de prevención y control, como son las medidas de barrera, la higiene de manos y la limpieza y desinfección (5). Además, los mecanismos de resistencia intrínsecos o adquiridos por la bacteria pueden llevar a la aparición de multirresistencia, siendo los bacilos Gram negativos uno de los grupos bacterianos más importantes (6).

De las bacterias Gram negativas, las Enterobacteriaceae, Pseudomonas aeruginosa y Acinetobacter baumannii son agentes causales de múltiples infecciones (7), y su capacidad de diseminación se considera incluso mayor que la de los cocos Gram positivos (8). La resistencia antimicrobiana de los bacilos Gram negativos multirresistentes está determinada no sólo por mutaciones cromosómicas sino también por la adquisición de genes transferibles entre diferentes especies. El mecanismo de resistencia más importante de las bacterias Gram negativas es la producción de betalactamasas; clínicamente se considera que las de mayor impacto son las betalactamasas de espectro extendido (BLEE), las betalactamasas

\section{Correspondencia:}

María Virginia Villegas, Carrera 125 № 19-225, Cali, Colombia Teléfono: (572) 555 2164; fax: (572) 5552638

mariavirginia.villegas@gmail.com

Recibido: 20/05/13; aceptado: 17/10/13 inducibles tipo Amp-C y las carbapenemasas (9). El aislamiento de carbapenemasas en Enterobacteriaceae, $P$. aeruginosa y $A$. baumannii se ha incrementado durante los últimos 10 años (10), siendo las más frecuentemente reportadas hasta el momento las carbapenemasas del grupo A, como la KPC (Klebsiella pneumoniae carbapenemase), las del grupo B como la VIM (Verona Integron-encoded Metallo-betalactamase) y la IMP (imipenemasa), así como las del grupo D, el cual está conformado por las OXA (oxacilinasas) (11). Entre los mecanismos no enzimáticos se destacan la alteración de los transportadores de membrana de tipo porina y la expresión de las bombas de expulsión $(12,13)$.

Los bacilos Gram negativos multirresistentes han causado brotes hospitalarios en todo el mundo (14), y han sido identificados, además, como colonizadores y contaminantes de pacientes, de trabajadores de la salud, así como del medio ambiente dentro y fuera de los hospitales (15). Su creciente prevalencia en la última década ha ameritado la implementación de sistemas de vigilancia epidemiológica como uno de los métodos más efectivos para monitorizar los cambios en la sensibilidad de cualquier microorganismo $(16,17)$.

La vigilancia epidemiológica a través del software WHONET, desarrollado por la Organización Mundial de la Salud (OMS), permite el estudio fenotípico de los perfiles de resistencia antimicrobiana (18); añadido a esto, el uso de herramientas moleculares para el estudio genético y evolutivo de los microorganismos multirresistentes (19) podría ayudar a determinar la magnitud del fenómeno, a desarrollar estrategias dirigidas a disminuir la transmisión entre pacientes, a implementar programas para el uso adecuado de antimicrobianos y a prevenir el número de pacientes infectados y colonizados por bacterias multirresistentes (20).

Este estudio se propuso describir la evolución de los perfiles fenotípicos y moleculares de los bacilos Gram negativos durante el periodo entre 2009 y 2012 en 23 hospitales de alta complejidad de Colombia. 


\section{Materiales y métodos}

\section{Diseño del estudio}

Se llevó a cabo un estudio descriptivo de vigilancia fenotípica y molecular de la resistencia antimicrobiana de bacilos Gram negativos aislados durante el periodo de enero de 2009 a diciembre de 2012 en unidades de cuidados intensivos de 23 clínicas y hospitales en Colombia. Se analizaron aislamientos de $K$. pneumoniae, E. coli, $P$. aeruginosa y $A$. baumannii obtenidos en las unidades de cuidados intensivos médico-quirúrgicas de adultos, pediátricas y neonatales de hospitales de alta complejidad (250-500 camas) en 10 ciudades colombianas, seis de ellos localizados en Bogotá, cuatro en Cali, tres en Medellín, tres en Bucaramanga, dos en Pasto y uno en cada una de las siguientes ciudades: Barranquilla, Cúcuta, Ibagué, Pereira y Neiva. Todos los hospitales pertenecen al Grupo para el Estudio de la Resistencia Nosocomial (sic.) en Colombia liderado por el Centro Internacional de Entrenamiento e Investigaciones Médicas (CIDEIM).

\section{Vigilancia fenotípica}

Para el análisis fenotípico se construyó una base de datos con los resultados de los perfiles de sensibilidad y de las pruebas fenotípicas para detección de betalactamasas de espectro extendido (BLEE) realizadas en cada una de las instituciones mediante sistemas automatizados. En 14 instituciones se utilizó Vitek ${ }^{\circledR} 2$, en seis, MicroScan ${ }^{\circledR}$ y en tres, Phoenix ${ }^{\circledR}$. Todos los laboratorios tenían control de calidad interno y externo. El control interno lo realizaron con cepas del ATCC (Escherichia coli ATCC $\AA$ 25922, Klebsiella pneumoniae ATCC $\AA$ 700603, Pseudomonas aeruginosa ATCC® 27853, Acinetobacter baumannii ATCC $\$$ 17978) y el externo estuvo a cargo del Instituto Nacional de Salud, PROASECAL, bioMérieux y Medical Laboratory Evaluation, entre otros.

La base de datos se procesó con el software WHONET, versión 5.6, previa estandarización y verificación de diccionarios por medio de BacLink. En el análisis se incluyó únicamente un aislamiento por paciente, correspondiente al primer bacilo Gram negativo aislado durante la hospitalización en la unidad de cuidados intensivos con pruebas de sensibilidad. Las pruebas de sensibilidad antimicrobiana se analizaron según las guías del Clinical and Laboratory Standards Institute (CLSI M100 S19) (21).

Se definieron como organismos resistentes a múltiples medicamentos aquellos que presentaran resistencia, al menos, a tres familias de anti- microbianos con actividad contra dicho patógeno (22). Para E. coli, el perfil de multirresistencia debía incluir, entre otros, resistencia a cefalosporinas de tercera y cuarta generación (23) y resistencia a carbapenémicos para K. pneumoniae, $P$. aeruginosa y $A$. baumannii $(9,24)$.

\section{Vigilancia de carbapenemasas por biología molecular}

Los laboratorios de microbiología de cada institución enviaron al Laboratorio de Microbiología y Biología Molecular del CIDEIM todos los aislamientos de bacilos Gram negativos con perfil fenotípico de resistencia al cefepime y, al menos, a un carbapenémico. La identificación y los perfiles de sensibilidad antimicrobiana fueron confirmados mediante el uso de la tecnología Vitek $^{\circledR} 2$ (bioMérieux, Marcy I’Etoile, France) y microdilución en caldo (Trek Diagnostic Systems, Westlake, $\mathrm{OH})$. Se realizó reacción en cadena de la polimerasa (PCR) a todas los aislamientos de $K$. pneumoniae, $P$. aeruginosa y $A$. baumannii como prueba confirmatoria para le detección de genes codificantes para las carbapenemasas KPC, VIM, IMP y OXA.

\section{Análisis estadístico}

Se hicieron análisis de tendencia según la frecuencia de aislamientos y por los tipo de muestra: sangre, orina, secreciones respiratorias y otros (secreciones de herida quirúrgica, piel y tejidos blandos, colecciones y abscesos), así como perfiles de sensibilidad por marcadores de resistencia $-E$. coli y K. pneumoniae (ceftazidime - CAZ, ceftriaxona - CRO, cefotaxime - CTX y ciprofloxacina - CIP, piperacilina-tazobactam - TZP, ertapenem - ETP, imipenem - IPM y meropenem - MEM), P. aeruginosa y A. baumannii (CIP-FEP-TZP-IMP-MEM), y perfiles fenotípicos de multirresistencia.

Para el análisis estadístico de los datos se calcularon y compararon los porcentajes de frecuencias y resistencias durante los años 2009, 2010, 2011 y 2012 para E. coli, K. pneumoniae, P. aeruginosa y $A$. baumannii, y se determinó su tendencia por medio de la prueba estadística de ji al cuadrado de tendencia lineal, con un nivel de significación estimado de 0,05. El software estadístico utilizado para los análisis fue Epidat, versión 3.1.

\section{Resultados}

De 38.048 microorganismos aislados en los últimos cuatro años, 24.203 (63 \%) correspondieron a bacilos Gram negativos; de ellos, se identificaron 
5.637 de E. coli, 5.302 de K. pneumoniae, 3.647 de $P$. aeruginosa y 1.525 de $A$. baumannii (figura 1). En general, $E$. coli fue el microorganismo más frecuentemente aislado con un promedio anual de 14,8 \%; sin embargo, el número de aislamientos de $K$. pneumoniae aumentó significativamente $(p<0,001)$ hasta ser el microorganismo más frecuente para el año 2012 con $15 \%$ de los casos. $P$. aeruginosa presentó un comportamiento anual estable con $9,7 \%$ de los casos, mientras que $A$. baumannii disminuyó de 5,1\% en el 2009 a 3,4 \% en el $2012(p<0,001)$.

En la distribución anual de los aislamientos según el tipo de muestra (cuadro 1), se observó un aumento estadísticamente significativo en todos los microorganismos aislados en secreciones respiratorias, llegando a ser el tipo de muestra más frecuente para $A$. baumannii y $P$. aeruginosa. Además, los aislamientos de E. coli y $P$. aeruginosa en orina aumentaron de 45 y $10 \%$ en el 2009 , a 50,4 y $16 \%$ en el 2012, respectivamente $(p=0,009$ y $p>0,001)$. Por el contrario, no se observaron cambios de tendencia en aislamientos en sangre, excepto en $K$. pneumoniae, el cual disminuyó de $28 \%$ en el año 2009 a 22,5 \% en el 2012 ( $p<0,001)$.

Los marcadores de resistencia (cuadro 2) muestran que $E$. coli, con un promedio de $16,3 \%$ de resistencia a cefalosporinas de tercera generación (Cef3ra), aumentó, al menos, en $1,5 \%$ su resistencia a CAZ, CRO y CTX en el 2012 y disminuyó el

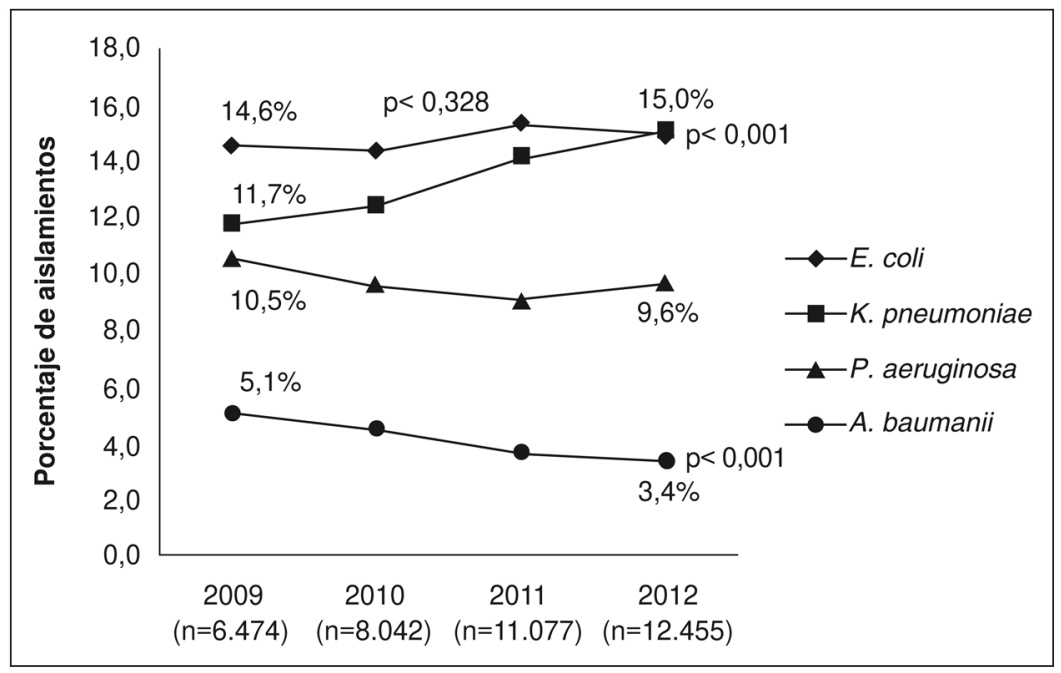

Figura 1. Tendencia de los aislamientos de Escherichia coli, Klebsiella pneumoniae y Pseudomonas aeruginosaen las unidades de cuidados intensivos de 23 clínicas y hospitales de la red de resistencia bacteriana nosocomial (sic.) del CIDEIM. Se calcula el porcentaje de aislamiento de cada microorganismo en relación con el número total de aislamientos en el mismo periodo de estudio.

Cuadro 1. Porcentaje de aislamientos por tipo de muestra de Escherichia coli, Klebsiella pneumoniae, Pseudomonas aeruginosa y Acinetobacter baumannii en las unidades de cuidados intensivos de 23 clínicas y hospitales, 2009 a 2012.

\begin{tabular}{|c|c|c|c|c|c|}
\hline & 2009 (\%) & $2010(\%)$ & 2011 (\%) & 2012 (\%) & $\mathbf{p}$ \\
\hline Escherichia coli & $n=937$ & $n=1.150$ & $n=1.693$ & $\mathrm{n}=1.845$ & \\
\hline Sangre & 13,7 & 14,0 & 13,3 & 13,3 & 0,671 \\
\hline Orina & 44,9 & 49,1 & 51,3 & 50,4 & 0,0088 \\
\hline Secreciones respiratorias & 3,1 & 3,5 & 4,4 & 6,5 & 0,0000 \\
\hline Otros & 36,2 & 34,3 & 34,4 & 31,8 & - \\
\hline Klebsiella pneumoniae & $\mathrm{n}=861$ & $\mathrm{n}=995$ & $\mathrm{n}=1.557$ & $\mathrm{n}=1.864$ & \\
\hline Sangre & 28,0 & 27,7 & 25,4 & 22,5 & 0,0003 \\
\hline Orina & 19,0 & 21,3 & 23,0 & 21,8 & 0,1198 \\
\hline Secreciones respiratorias & 10,6 & 10,2 & 18,0 & 19,4 & 0,0000 \\
\hline Otros & 38,6 & 37,1 & 32,4 & 35,5 & - \\
\hline Pseudomonas aeruginosa & $n=673$ & $n=763$ & $n=998$ & $n=1.197$ & \\
\hline Sangre & 20,4 & 21,5 & 20,1 & 19,6 & 0,5037 \\
\hline Orina & 10,0 & 12,7 & 16,6 & 16,1 & 0,0001 \\
\hline Secreciones respiratorias & 12,3 & 15,1 & 19,5 & 23,0 & 0,0000 \\
\hline Otros & 39,4 & 34,9 & 25,5 & 26,3 & - \\
\hline Acinetobacter baumannii & $n=323$ & $\mathrm{n}=360$ & $\mathrm{n}=407$ & $\mathrm{n}=424$ & \\
\hline Sangre & 35,9 & 38,6 & 44,0 & 32,3 & 0,4871 \\
\hline Orina & 4,0 & 5,6 & 6,1 & 5,7 & 0,3285 \\
\hline Secreciones respiratorias & 17,0 & 18,9 & 20,6 & 40,3 & 0,0000 \\
\hline Otros & 39,3 & 33,9 & 27,8 & 24,1 & - \\
\hline
\end{tabular}


Cuadro 2. Porcentaje de resistencia de Escherichia coli, Klebsiella pneumoniae, Pseudomonas aeruginosa y Acinetobacter baumannii en las unidades de cuidados intensivos de 23 clínicas y hospitales de la red de resistencia bacteriana nosocomial (sic.) del CIDEIM, 2009 a 2012.

\begin{tabular}{|c|c|c|c|c|c|}
\hline & 2009 (\%) & $2010(\%)$ & $2011(\%)$ & $2012(\%)$ & p \\
\hline Escherichia coli & $\mathrm{n}=947$ & $\mathrm{n}=1.130$ & $n=1650$ & $\mathrm{n}=1.868$ & \\
\hline Ceftazidime & 16,7 & 14,7 & 15,2 & 18,6 & 0,0594 \\
\hline Ceftriaxona & 17,0 & 16,6 & 15,2 & 18,6 & 0,2861 \\
\hline Cefotaxime & 15,8 & 15,1 & 15,6 & 16,9 & 0,4554 \\
\hline Ciprofloxacina & 34,5 & 30,6 & 28,5 & 31,7 & 0,1968 \\
\hline Cefepime & 15,2 & 14,9 & 13,1 & 17,1 & 0,1786 \\
\hline Piperacilina-tazobactam & 13,3 & 14,7 & 14,1 & 13,4 & 0,7751 \\
\hline Ertapenem & 0,4 & 0,8 & 0,9 & 1,2 & 0,0719 \\
\hline Imipenem & 0,1 & 0,2 & 0,4 & 0,4 & 0,1248 \\
\hline Meropenem & 0,2 & 0,2 & 0,2 & 0,3 & 0,3682 \\
\hline Klebsiella pneumoniae & $n=865$ & $n=973$ & $\mathrm{n}=1.464$ & $\mathrm{n}=1.915$ & \\
\hline Ceftazidime & 30,2 & 33,2 & 29,6 & 33,9 & 0,1443 \\
\hline Ceftriaxona & 32,4 & 34,7 & 31,9 & 34,7 & 0,412 \\
\hline Cefotaxime & 32,8 & 37,8 & 30,5 & 36,3 & 0,1953 \\
\hline Ciprofloxacina & 17,7 & 19,1 & 19,8 & 23,9 & 0,0001 \\
\hline Cefepime & 12,8 & 30,8 & 32,8 & 29,7 & 0,1433 \\
\hline Piperacilina-tazobactam & 30,5 & 34,7 & 30,2 & 31,8 & 0,8315 \\
\hline Ertapenem & 7,1 & 8,7 & 9,0 & 12,8 & 0,0000 \\
\hline Imipenem & 1,5 & 6,4 & 5,6 & 8,9 & 0,0000 \\
\hline Meropenem & 1,3 & 5,9 & 5,6 & 9,1 & 0,0000 \\
\hline Pseudomonas aeruginosa & $n=675$ & $n=751$ & $n=961$ & $n=1.120$ & \\
\hline Ciprofloxacina & 24,6 & 22,5 & 29,6 & 28,8 & 0,0033 \\
\hline Cefepime & 23,2 & 23,0 & 36,4 & 32,0 & 0,0000 \\
\hline Piperacilina-tazobactam & 29,4 & 31,0 & 39,0 & 35,9 & 0,0004 \\
\hline Imipenem & 23,7 & 27,9 & 35,8 & 34,5 & 0,0000 \\
\hline Meropenem & 22,0 & 24,8 & 32,5 & 30,5 & 0,0000 \\
\hline Acinetobacter baumannii & $\mathrm{n}=325$ & $\mathrm{n}=356$ & $n=388$ & $n=438$ & \\
\hline Ciprofloxacina & 65,5 & 65,2 & 55,9 & 64,4 & 0,3357 \\
\hline Cefepime & 60,8 & 68,8 & 60,7 & 61,6 & 0,779 \\
\hline Piperacilina-tazobactam & 52,3 & 71,8 & 68,5 & 72,3 & - \\
\hline Imipenem & 57,8 & 35,8 & 39,5 & 37,1 & 0,0001 \\
\hline Meropenem & 56,0 & 60,5 & 96,0 & 98,8 & 0,0000 \\
\hline
\end{tabular}

*Los datos de 2011-2012 fueron obtenidos de 15 instituciones de la red de resistencia bacteriana nosocomial (sic.) del CIDEIM, las ocho instituciones restantes combinaron diferentes metodologías.

número de casos resistentes a CIP de 34,5 a $31,7 \%$. K. pneumoniae incrementó la resistencia a carbapenémicos en un promedio de $7 \%$, alcanzando $12,8 \%$ de resistencia a ETP en el $2012(p<0,001)$. $P$. aeruginosa alcanzó un promedio de $28,7 \%$ de resistencia a FEP y de $30,5 \%$ a IMP, siendo el antibiótico más afectado. A. baumannii presentó una disminución de 20,7 \% (57,8 a 37,1\%) en la resistencia a IMP $(p<0,001)$, pero un aumento de $42,8 \%$ frente a MEM $(56,0$ a 98,8 \%) $(p<0,001)$.

En cuanto al análisis de fenotipos, E. coli, con resultado positivo en la prueba de BLEE, representó $14,7 \%(599 / 4.148)$ del total de aislamientos. El perfil de multirresistencia de E. coli (figura 2) se expresó en la resistencia a las cefalosporinas de tercera y cuarta generación (BLEE +), a CIP y TZP, con un aumento de $61,9 \%$ en el 2009 a $79,1 \%$ en el 2012 ( $p<0,001)$. Igualmente, se observó un incremento de 5,6 \% (1,3 a 6,9 \%; $\mathrm{p}<0,001)$ en el perfil de multirresistencia de $K$. pneumoniae, constituido por su resistencia a los carbapenémicos y también a las cefalosporinas de tercera generación y a CIP (figura 3).
Durante la vigilancia molecular de los bacilos Gram negativos, se captaron 1.248 cepas distribuidas en $590(47,2 \%)$ de $P$. aeruginosa, $417(33,4 \%)$ de $K$. pneumoniae y 241 (19,3\%) de A. baumannii. La carbapenemasa más frecuente en $P$. aeruginosa fue la VIM, con 46,5\% (105 de 226 cepas estudiadas). En K. pneumoniae fue la KPC, con $68,4 \%$ de los casos (154/225) (figura 3). En $A$. baumannii, fue la OXA-23, con 97,7 \% de los casos (168/172). En todas las ciudades incluidas en el estudio se observó KPC en K. pneumoniae, mientras que en seis de nueve ciudades se encontró, además, presencia de VIM y KPC en $P$. aeruginosa y OXA23 en $A$. baumannii (figura 4).

\section{Discusión}

Los bacilos Gram negativos continúan siendo los microorganismos más frecuentemente aislados en las unidades de cuidados intensivos, tal como se viene reportando desde el año 2003 a través de la vigilancia epidemiológica realizada por nuestro Grupo para el Estudio de la Resistencia Nosocomial (sic.) en Colombia $(25,26)$. 
Escherichia coli, K. pneumoniae, $P$. aeruginosa y $A$. baumannii lideran el número de aislamientos entre los bacilos Gram negativos, tal como lo ha evidenciado el programa SMART en Estados Unidos para el periodo 2009-2011 (27) y el estudio TEST en el este europeo entre 2004 y 2010 (17); sin embargo, durante este periodo de vigilancia se logró detectar un aumento del número de aislamientos de $K$. pneumoniae, que incluso superó a E. coli en el 2012. Durante el periodo de estudio se encontró un aumento significativo de las bacterias estudiadas en muestras de tracto respiratorio (aspirados orotraqueales), hallazgos que deberán corroborarse con estudios clínicos que permitan determinar el rol de los bacilos Gram negativos multirresistentes en infecciones respiratorias.

Escherichia coli, con un fenotipo sugestivo de BLEE de $16 \%$ por su resistencia a cefalosporinas de tercera generación y a FEP, presentó un comportamiento semejante a lo reportado en las unidades de cuidados intensivos de los países europeos (28); por el contrario, el estudio SENTRY, realizado en cuatro países latinoamericanos,

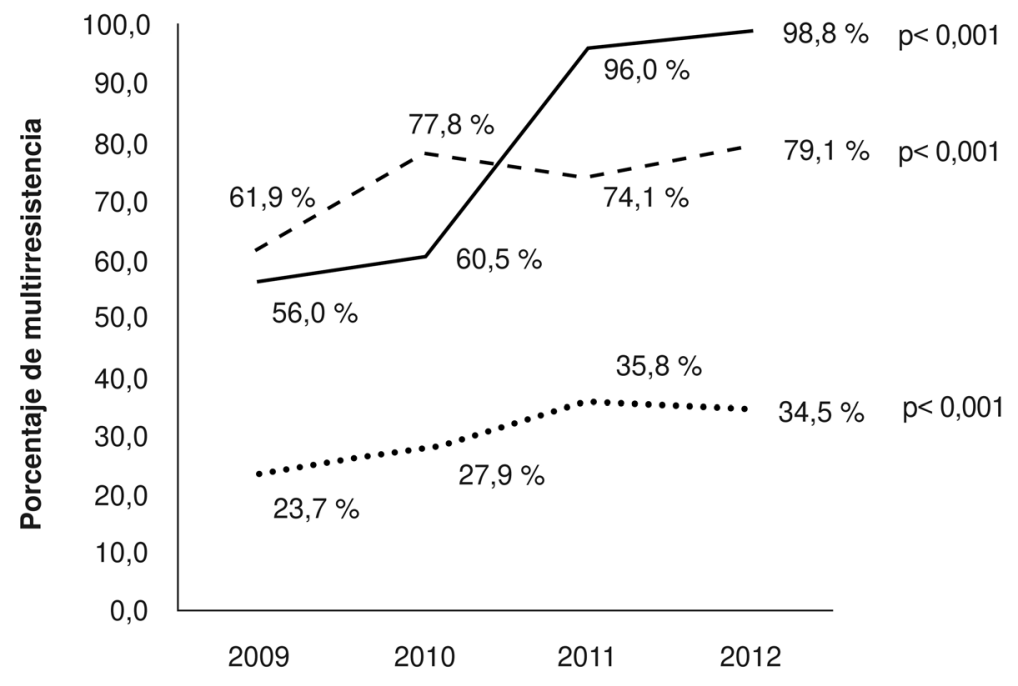

Figura 2. Tendencias en la frecuencia de las bacterias multirresistentes. Escherichia coli positivo para BLEE y resistente a ciprofloxacina; Pseudomonas aeruginosa y Acinetobacter baumannii resistentes a carbapenem en las unidades de cuidados intensivos de 23 clínicas y hospitales de la red de resistencia bacteriana nosocomial (sic.) del CIDEIM. Se calcula el porcentaje de aislamiento de cada microorganismo en relación con el número total de aislamientos en el mismo periodo de estudio.

Figura 3. Tendencias en la frecuencia de Klebsiella pneumoniae resistente a carbapenémicos y $K$. pneumoniae $\mathrm{KPC}+$ en las unidades de cuidados intensivos de clínicas y hospitales de la red de resistencia bacteriana nosocomial (sic.) del CIDEIM. Se calcula el porcentaje de aislamiento de cada microorganismo en relación con el número total de aislamientos en el mismo periodo de estudio.

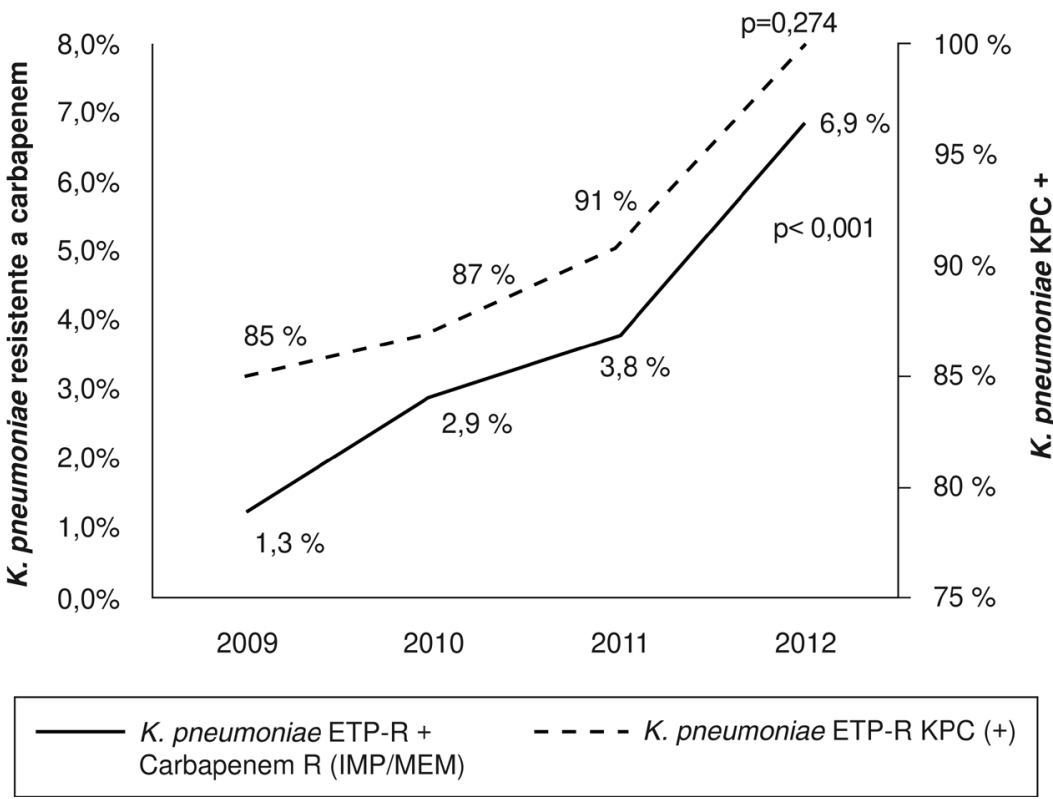




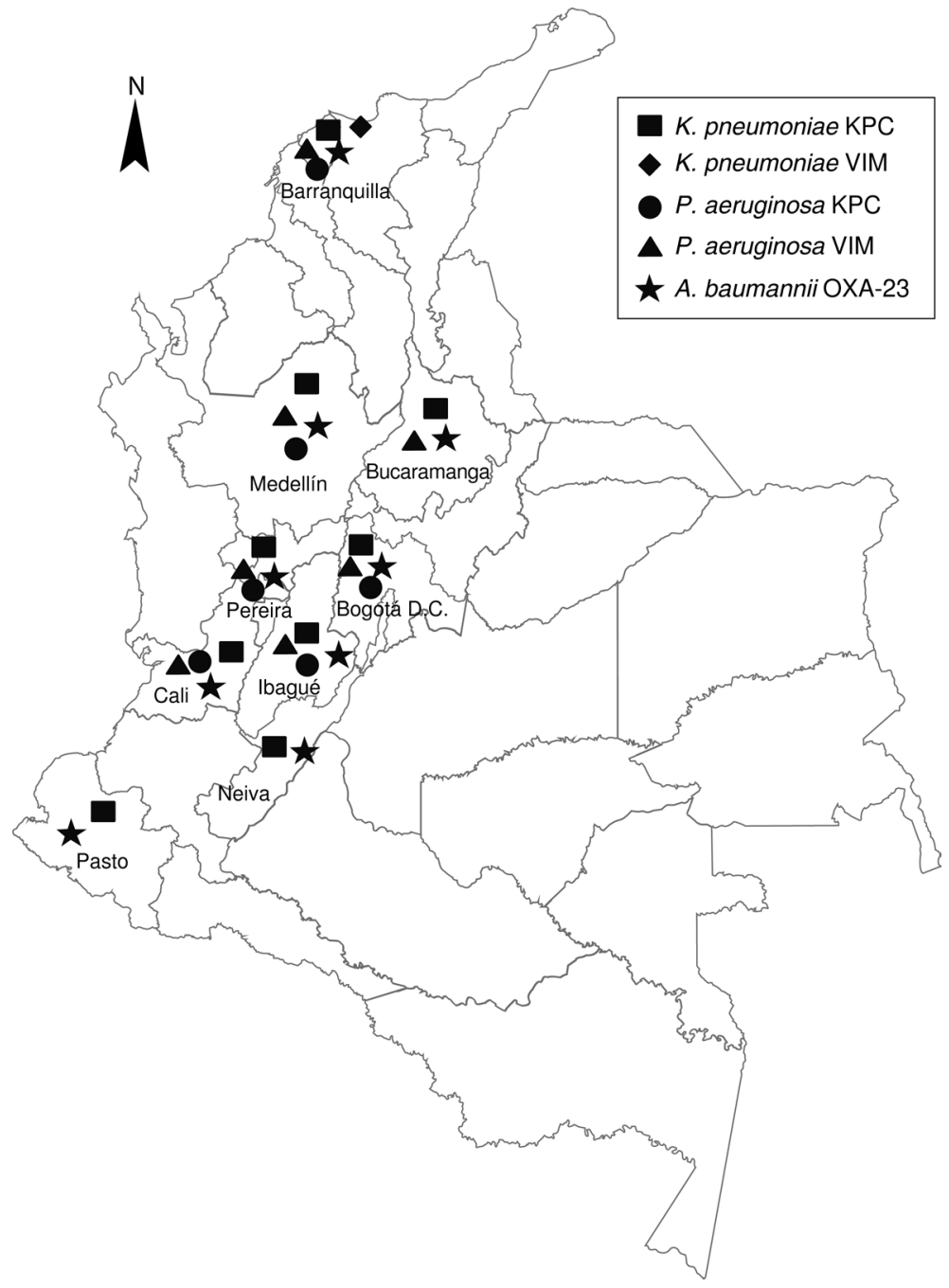

Figura 4. Presencia de carbapenemasas en nueve ciudades capitales de Colombia distribuidas por tipo de microorganismo y carbapenemasa asociada. reportó un mayor porcentaje de fenotipos sugestivos de BLEE, alcanzando hasta $23,9 \%$ para las cefalosporinas de tercera generación (29).

Por otro lado, nuestros datos revelaron un incremento de la resistencia a carbapenémicos en $K$. pneumoniae; este hallazgo es similar a lo descrito por Kaiser, et al., en el estudio SENTRY 2007-2009 en hospitales de Estados Unidos (30). Los hallazgos moleculares de las cepas de $K$. pneumoniae resistentes a carbapenem corroboraron la presencia y el aumento de blaKPC durante el periodo de estudio, fenómeno que ha sido reportado a nivel mundial (24). En Colombia, la presencia de KPC se ha descrito en varias ciudades del país, y su diseminación se ha explicado por la presencia de elementos genéticos móviles, los cuales han sido reportados en clones internacionales reconocidos como exitosos por su capacidad de propagación entre otras especies bacterianas (30-33).
Pseudomonas aeruginosa, con un perfil de multirresistencia de $34,5 \%$ en el 2012, presentó un comportamiento semejante a lo reportado por Bertrand, et al., en un análisis conglomerado del comportamiento en Norteamérica y Suramérica, Europa, Medio Oriente y el Pacífico asiático (34). En Colombia se ha descrito la presencia de VIM-2 en $P$. aeruginosa en diferentes ciudades (35), de forma similar a lo reportado por Amudhan, et al., en infecciones hospitalarias de unidades de cuidados intensivos en India (36); además, se ha encontrado la presencia de VIM y KPC de forma simultánea en $P$. aeruginosa (37).

Acinetobacter baumannii conserva un perfil fenotípico de multirresistencia igual a lo reportado por Kempf, et al. (38), presentando un incremento en la resistencia a carbapenémicos a lo largo de los años y alcanzando niveles de resistencia semejantes a los reportados en países europeos 
como Turquía, Grecia, Italia, España e Inglaterra, que oscilan entre 50 y $70 \%$ de multirresistencia (39). La OXA-23 en A. baumannii fue la carbapenemasa más frecuentemente detectada en el país; esta enzima contribuye al perfil de multirresistencia, el cual podría estar incrementado por la presencia de brotes hospitalarios (40-42).

En conclusión, se observó una tendencia al incremento de bacilos Gram negativos multirresistentes y una amplia distribución de las carbapenemasas en Colombia, especialmente KPC, VIM y OXA-23. La articulación de la biología molecular a los sistemas de vigilancia epidemiológica tradicionales permitió el análisis integral de la resistencia; estos hallazgos podrían servir para el desarrollo de programas de uso adecuado de antimicrobianos y la elaboración de guías para la prevención y contención de bacterias multirresistentes en Colombia.

\section{Agradecimientos}

A los miembros del Grupo de Resistencia Bacteriana Nosocomial en Colombia, por su apoyo y compromiso: en Cali, Ernesto Martínez, Christian Pallares, Fernando Rosso, Juan Diego Vélez, Claudia Castañeda, Martín Muñoz, Beatriz Vanegas y Lorena Matta; en Medellín, Paola Rojas, Walter Zea, Jorge Nagles, Luz Teresita Correa, Ana Lucía Correa y Ana María Bedoya; en Bogotá, Henry Mendoza, Martha Patricia Meléndez, Gerson Arias, Henry Oliveros, Carlos Pérez, María Nilse González, Sandra Gualtero, Sandra Valderrama, Carlos Álvarez, Guillermo Prada y Clara Luz Rico; en Bucaramanga, Beatriz López, Claudia Bárcenas, Agustín Vega, Myriam Fanny Amaya y Edgar Bernal; en Barranquilla, Rubén Camargo y Adriana Marín; en Pereira, Carmen Elisa Llanos y Myriam Gómez; en Ibagué, Claudia Echeverri, Amparo Ovalle y María del Rosario Aldana; en Cúcuta, Luz Marina Osorio y Zulma Urbina; en Neiva, Johanna Osorio y Jorge Ramos, y en Pasto, Marco Antonio Solarte, Rocío Ortega, Mónica Guerrero y Ana Milena Torres.

\section{Conflicto de intereses}

María Virginia Villegas ha recibido apoyo económico para investigación y ha sido asesora de Baxter, Merck Sharp \& Dohme, Pfizer, Merck S.A, Novartis, AstraZeneca y Janssen Cilag. Los otros autores no declaran conflicto de intereses.

\section{Financiación}

La conformación de la red de hospitales pertenecientes al Grupo para el Estudio de la Resistencia Nosocomial en Colombia recibe apoyo financiero de Baxter, Merck Sharp \& Dohme, Pfizer, Merck S.A, Novartis, AstraZeneca, bioMérieux, Bayer y Janssen Cilag.

\section{Referencias}

1. Kanafani ZA, Mehio-Sibai A, Araj GF, Kanaan M, Kanj SS. Epidemiology and risk factors for extended-spectrum beta-lactamase-producing organisms: A case-control study at a tertiary care center in Lebanon. Am J Infect Control. 2005;33:326-32. http://dx.doi.org/10.1016/j.ajic.2005.03.009

2. Tansarli GS, Karageorgopoulos DE, Kapaskelis A, Falagas ME. Impact of antimicrobial multidrug resistance on inpatient care cost: An evaluation of the evidence. Expert Rev Anti Infect Ther. 2013;11:321-31. http://dx.doi. org/10.1586/eri.13.4

3. Bhattacharya S. Early diagnosis of resistant pathogens: how can it improve antimicrobial treatment? Virulence. 2013;4:172-84. http://dx.doi.org/10.4161/viru.23326

4. Moreira MR, Guimarães MP, Rodrigues AA, Gontijo Filho PP. Antimicrobial use, incidence, etiology and resistance patterns in bacteria causing ventilator-associated pneumonia in a clinical-surgical intensive care unit. Rev Soc Bras Med Trop. 2013;46:39-44.

5. Bearman G, Rosato AE, Duane TM, Elam K, Sanogo $\mathrm{K}$, Haner C, et al. Trial of universal gloving with emollientimpregnated gloves to promote skin health and prevent the transmission of multidrug-resistant organisms in a surgical intensive care unit. Infect Control Hosp Epidemiol. 2010;31:491-7. http://dx.doi.org/10.1086/651671

6. Moellering RC Jr, Graybill JR, McGowan JE Jr, Corey L. Antimicrobial resistance prevention initiative--an update: Proceedings of an expert panel on resistance. Am J Med. 2007;35:S4-25. http://dx.doi.org/10.1016/j.ajic.2007.08.001

7. Thomson JM, Bonomo RA. The threat of antibiotic resistance in Gram-negative pathogenic bacteria: Betalactams in peril! Curr Opin Microbiol. 2005;8:518-24. http:// dx.doi.org/10.1016/j.mib.2005.08.014

8. Giamarellou H, Antoniadou A, Kanellakopoulou K. Acinetobacter baumannii: A universal threat to public health? Int J Antimicrob Agents. 2008;32:106-19. http://dx. doi.org/10.1016/j.ijantimicag.2008.02.013

9. Paterson DL. Resistance in gram-negative bacteria: Enterobacteriaceae. Am J Med. 2006;119(Suppl.1):S20-8. http://dx.doi.org/10.1016/j.amjmed.2006.03.013

10. Nordmann P, Naas T, Poirel L. Global spread of carbapenemase-producing Enterobacteriaceae. Emerg Infect Dis. 2011;17:1791-8. http://dx.doi.org/10.3201/eid1710.110655

11. Queenan AM, Bush K. Carbapenemases: The versatile beta-lactamases. Clin Microbiol Rev. 2007;20:440-458. http://dx.doi.org/10.1128/CMR.00001-07

12. Mérens A, Delacour H, Plésiat $P$, Cavallo JD, Jeannot $K$. Pseudomonas aeruginosa et résistance aux antibiotiques. Revue Francophone des Laboratoires. 2011:49-62. http://dx. doi.org/10.1016/S1773-035X (11)71102-9

13. Gordon NC, Wareham DW. Multidrug-resistant Acinetobacter baumannii: Mechanisms of virulence and resistance. Int J Antimicrob Agents. 2010;35:219-26. http://dx.doi.org/10. 1016/j.ijantimicag.2009.10.024 
14. Bilavsky E, Pfeffer I, Tarabeia J, Schechner V, Abu-Hanna J, Grisaru-Soen G, et al. Outbreak of multidrug-resistant Pseudomonas aeruginosa infection following urodynamic studies traced to contaminated transducer. J Hosp Infect. 2013;83:344-6. http://dx.doi.org/10.1016/j.jhin.2013.01.003

15. Vento TJ, Cole DW, Mende K, Calvano TP, Rini EA, Tully CC, et al. Multidrug-resistant gram-negative bacteria colonization of healthy US military personnel in the US and Afghanistan. BMC Infect Dis. 2013;13:68. http://dx.doi.org/10. 1186/1471-2334-13-68.

16. Isturiz R. Global resistance trends and the potential impact on empirical therapy. Int J Antimicrob Agents. 2008;32(Suppl.4):S201-6. http://dx.doi.org/10.1016/S09248579(09)70003-2

17. Balode A, Punda-Polić V, Dowzicky MJ. Antimicrobial susceptibility of Gram-negative and Gram-positive bacteria collected from countries in Eastern Europe: Results from the Tigecycline Evaluation and Surveillance Trial (TEST) 2004-2010. Int J Antimicrob Agents. 2013;41:527-35. http:// dx.doi.org/10.1016/j.jjantimicag.2013.02.022

18. Agarwal A, Kapila K, Kumar S. WHONET software for the surveillance of antimicrobial susceptibility. Med J Armed Forces India. 2009;65:264-6. http://dx.doi.org/10.1016/ S0377-1237(09)80020-8

19. Sangal V, Girvan EK, Jadhav S, Lawes T, Robb A, Vali $\mathrm{L}$, et al. Impacts of a long-term programme of active surveillance and chlorhexidine baths on the clinical and molecular epidemiology of meticillin-resistant Staphylococcus aureus (MRSA) in an intensive care unit in Scotland. Int $\mathrm{J}$ Antimicrob Agents. 2012;40:323-31. http://dx.doi.org/10. 1016/j.jjantimicag.2012.06.007

20. D'Agata EMC, Horn MA, Ruan S, Webb GF, Wares JR. Efficacy of infection control interventions in reducing the spread of multidrug-resistant organisms in the hospital setting. Plos One. 2012;7:e30170. http://dx.doi.org/10.1371/ journal.pone. 0030170

21. Clinical and Laboratory Standards Institute. Performance Standards for Antimicrobial Susceptibility Testing; Nineteenth Informational Supplement. CLSI document M100-S19. Wayne, PA: Clinical and Laboratory Standards Institute; 2009.

22. Alekshun MN, Levy SB. Molecular mechanisms of antibacterial multidrug resistance. Cell. 2007;128:1037-50. http://dx.doi.org/10.1016/j.cell.2007.03.004

23. Picozzi S, Ricci C, Gaeta M, Macchi A, Dinang E, Paola G, et al. Do we really know the prevalence of multi-drug resistant Escherichia coli in the territorial and nosocomial population? Urol Ann. 2013;5:25-9. http://dx.doi.org/10. 4103/0974-7796.106962

24. Chen LF, Anderson DJ, Paterson DL. Overview of the epidemiology and the threat of Klebsiella pneumoniae carbapenemases (KPC) resistance. Infect Drug Resist. 2012;5:133-41. http://dx.doi.org/10.4103/0974-7796.106962

25. Miranda MC, Pérez F, Zuluaga T, Olivera $\mathbf{M}$ del $\mathbf{R}$, Correa A, Reyes SL, et al. Antimicrobial resistance in gram negative bacteria isolated from intensive care units of Colombian hospitals, WHONET 2003, 2004 and 2005. Biomédica. 2006;26:424-33.

26. Briceño DF, Correa A, Valencia C, Torres JA, Pacheco $\mathbf{R}$, Montealegre MC, et al. Antimicrobial resistance of
Gram negative bacilli isolated from tertiary-care hospitals in Colombia. Biomédica. 2010;30:371-81.

27. Bouchillon SK, Badal RE, Hoban DJ, Hawser SP. Antimicrobial susceptibility of inpatient urinary tract isolates of Gram-negative bacilli in the United States: Results from the Study for Monitoring Antimicrobial Resistance Trends (SMART) Program: 2009-2011. Clin Ther. 2013;35:872-7. http://dx.doi.org/10.1016/j.clinthera.2013.03.022

28. Meyer E, Gastmeier P, Deja M, Schwab F. Antibiotic consumption and resistance: Data from Europe and Germany. Int J Med Microbiol. 2013;303:388-95. http://dx. doi.org/10.1016/j.jmm.2013.04.004

29. Gales AC, Castanheira M, Jones RN, Sader HS. Antimicrobial resistance among Gram-negative bacilli isolated from Latin America: Results from SENTRY Antimicrobial Surveillance Program (Latin America, 20082010). Diagn Microbiol Infect Dis. 2012;73:354-60. http:// dx.doi.org/10.1016/j.diagmicrobio.2012.04.007

30. Kaiser RM, Castanheira M, Jones RN, Tenover F, Lynfield R. Trends in Klebsiella pneumoniae carbapenemasepositive K. pneumoniae in US hospitals: Report from the 2007-2009 SENTRY Antimicrobial Surveillance Program. Diagn Microbiol Infect Dis. 2013;76:356-60.http://dx.doi. org/10.1016/j.diagmicrobio.2013.03.032

31. Cuzon G, Naas T, Correa A, Quinn JP, Villegas MV, Nordmann P. Dissemination of the KPC-2 carbapenemase in non-Klebsiella pneumoniae enterobacterial isolates from Colombia. Int J Antimicrob Agents. 2013;42:59-62. http:// dx.doi.org/10.1016/j.ijantimicag.2013.04.002

32. Mojica MF, Correa A, Vargas DA, Maya JJ, Montealegre MC, Rojas LJ, et al. Molecular correlates of the spread of KPC-producing Enterobacteriaceae in Colombia. Int $\mathrm{J}$ Antimicrob Agents. 2012;40:277-9. http://dx.doi.org/10.1016/j. ijantimicag.2012.05.006

33. Villegas MV, Lolans K, Correa A, Kattan JN, López JA, Quinn JP. First identification of Pseudomonas aeruginosa isolates producing a KPC-type carbapenemhydrolyzing beta-lactamase. Antimicrob Agents Chemother. 2007;51:1553-5. http://dx.doi.org/10.1128/AAC.01405-06

34. Bertrand X, Dowzicky MJ. Antimicrobial susceptibility among gram-negative isolates collected from intensive care units in North America, Europe, the Asia-Pacific Rim, Latin America, the Middle East, and Africa between 2004 and 2009 as part of the Tigecycline Evaluation and Surveillance Trial. Clin Ther. 2012;34:124-37. http://dx.doi.org/10.1016/j. clinthera.2011.11.023

35. Villegas MV, Lolans K, del Rosario Olivera M, Suárez CJ, Correa A, Queenan AM, et al. First detection of metallo-betalactamase VIM-2 in Pseudomonas aeruginosa isolates from Colombia. Antimicrob Agents Chemother. 2006;50:226-9. http://dx.doi.org/10.1128/AAC.50.1.226-229.2006

36. Amudhan MS, Sekar U, Kamalanathan A, Balaraman S. blaIMP and blaVIM mediated carbapenem resistance in Pseudomonas and Acinetobacter species in India. J Infect Dev Ctries. 2012;26;6:757-62. http://dx.doi.org/10.3855/jidc. 2268

37. Correa A, Montealegre MC, Mojica MF, Maya JJ, Rojas LJ, De La Cadena EP, et al. First report of a Pseudomonas aeruginosa isolate coharboring KPC and VIM carbapenemases. Antimicrob Agents Chemother. 2012; 56:5422-3. http://dx.doi.org/10.1128/AAC.00695-12 
38. Kempf M, Rolain JM. Emergence of resistance to carbapenems in Acinetobacter baumannii in Europe: Clinical impact and therapeutic options. Int $\mathrm{J}$ Antimicrob Agents. 2012;39:105-14. http://dx.doi.org/10.1016/j.ijantimicag. 2011.10.004

39. Kresken M, Becker K, Seifert H, Leitner E, Körber-Irrgang B, Von Eiff C, et al. Resistance trends and in vitro activity of tigecycline and 17 other antimicrobial agents against Grampositive and Gram-negative organisms, including multidrugresistant pathogens, in Germany. Eur J Clin Microbiol Infect Dis. 2011;30:1095-103. http://dx.doi.org/10.1007/s10096011-1197-y

40. Villegas MV, Kattan JN, Correa A, Lolans K, Guzmán AM, Woodford N, et al. Dissemination of Acinetobacter baumannii clones with OXA-23 carbapenemase in Colombian hospitals. Antimicrob Agents Chemother. 2007;51:2001-4. http://dx.doi.org/10.1128/AAC.00226-07

41. D’Arezzo S, Principe L, Capone A, Petrosillo N, Petrucca A, Visca P. Changing carbapenemase gene pattern in an epidemic multidrug-resistant Acinetobacter baumannii lineage causing multiple outbreaks in central Italy. J Antimicrob Chemother. 2011;66:54-61. http://dx.doi. org/10.1093/jac/dkq407

42. Yang HY, Lee HJ, Suh JT, Lee KM. Outbreaks of imipenem resistant Acinetobacter baumannii producing OXA-23 beta-lactamase in a tertiary care hospital in Korea. Yonsei Med J. 2009;50:764-70. http://dx.doi.org/10.3349/ ymj.2009.50.6.764 